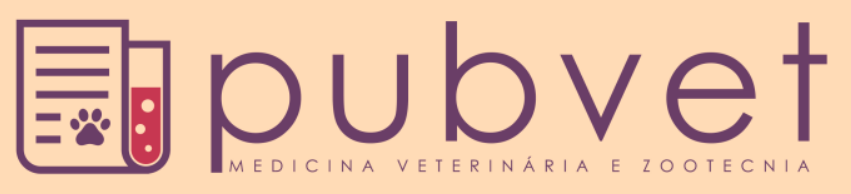

https://doi.org/10.31533/pubvet.v12n8a151.1-4

\title{
Produtividade de mandioca avaliada sobre adubação fosfatada e a adubação de cobertura
}

\author{
Alandionys Gonçalves de Lima ${ }^{1}$, Lucas Roberto de Carvalho $^{2}{ }^{\ominus}$, Marília Constantino Mota ${ }^{1}{ }^{\theta}$, \\ Antônio Florentino de Lima Junior ${ }^{2} \theta$, Juracy Mendes Moreira ${ }^{2} \theta$, Arinaldo Pereira da Silva ${ }^{2}{ }^{\circ}$, \\ Roberto Barbuio ${ }^{2}$, Juliano Queiroz Santana Rosa ${ }^{20 *}$
}

${ }^{I}$ Engenheiro Agronômico,

${ }^{2}$ Prof. Faculdade Montes Belos. São Luiz de Montes Belos - GO. E-mail: juraci.moreira@fmb.edu.br

*Autor para correspondência, E-mail: julianoqsr@gmail.com

\begin{abstract}
RESUMO. A mandioca é uma das mais promissoras culturas, na agricultura familiar, contribuindo no desenvolvimento social e econômico do Brasil. Apesar disso, tradicionalmente a cultura é conduzida com baixo investimento financeiro, geralmente, não havendo aplicação de corretivos e fertilizantes em doses adequadas. O objetivo deste trabalho foi avaliar o efeito de diferentes doses de adubação fosfatada e a adubação de cobertura na produtividade da mandioca (IAC 12). O experimento foi desenvolvido em parcela subdividida com 3 repetições. Avaliaram-se quatro doses de fósforo $(0 ; 40 ; 80$; $160 \mathrm{~kg}$ de $\mathrm{P}_{2} \mathrm{O}_{5} / \mathrm{ha}$ ) e a adubação de cobertura ( 0 e $250 \mathrm{~kg} / \mathrm{ha}$ de $\left.20-00-20\right)$. A colheita foi realizada 10 meses após o plantio e foi determinada a produtividade (kg/planta). Foi realizada análise de variância e teste de Scott-Knott para comparação de médias. Verificouse que houve resposta crescente à adubação fosfatada; a produtividade foi maior para a dose de $160 \mathrm{~kg}$ de $\mathrm{P}_{2} \mathrm{O}_{5} /$ ha, que é o dobro da dose máxima recomendada pela CFSEMG, demostrando que embora rústica, a planta é responsiva a adubação fosfatada. Com relação à adubação de cobertura, não houve diferença significativa entre os tratamentos. Concluísse que a cultivar de mandioca IAC 12 é responsiva a fósforo, havendo necessidade de estudos com maiores doses.
\end{abstract}

Palavras chave: Adubação de plantio, IAC 12, Manihot esculenta

\section{Yield of cassava evaluated on four levels of Phosphate fertilization and a cover fertilization}

ABSTRACT. Cassava is one of the most promising crops in family agriculture, contributing to the social and economic development of Brazil. In spite of this, traditionally the culture is conducted with low financial investment, generally, not having corrective and fertilizer application in adequate doses. The objective of this work was to evaluate the effect of different doses of phosphate fertilization and yield fertilization on cassava (IAC 12). The experiment was developed in a subdivided plot with 24 replicates. Four doses of phosphorus $\left(0,40,80,160 \mathrm{~kg}\right.$ of $\left.\mathrm{P}_{2} \mathrm{O}_{5} / \mathrm{ha}\right)$ and cover fertilization $(0$ and $250 \mathrm{~kg} / \mathrm{ha}$ of $20-$ -20-20) were evaluated. Harvesting was performed $\mathrm{xx}$ months after planting and productivity per plant was determined. Variance analysis and Scott-Knott's test were performed to compare means. There was an increasing response to phosphate fertilization; the productivity was higher for the $160 \mathrm{~kg}$ dose of $\mathrm{P} 2 \mathrm{O} 5$ / ha, which is twice the maximum dose recommended by CFSEMG, showing that although it is rustic, the plant is responsive to phosphate fertilization. Regarding the cover fertilization, there was no significant difference between the treatments. It was concluded that the cultivar of cassava IAC 12 is responsive to phosphorus, requiring studies with higher doses.

Keywords: planting fertilization, IAC 12, Manihot esculenta 


\title{
Efecto de la fertilización fosfatada y fertilización de cobertura sobre la productividad de la yuca
}

\begin{abstract}
RESUMEN. La yuca es uno de los cultivos más promisorios, en la agricultura familiar, contribuyendo al desarrollo social y económico de Brasil. A pesar de ello, tradicionalmente el cultivo se conduce con poca inversión financiera, generalmente, no habiendo aplicación de correctivos y fertilizantes a dosis adecuadas. El objetivo de este trabajo fue evaluar el efecto de diferentes dosis de fertilización fosfatada y la fertilización de cobertura en la productividad de la yuca (IAC 12). El experimento fue realizado en parcela subdividida con 3 repeticiones. Se evaluaron cuatro dosis de fósforo $(0,40,80,160 \mathrm{~kg}$ de P2O5/ha) y la fertilización de cobertura (0 y $250 \mathrm{~kg} /$ ha de 20-00-20). La cosecha se realizó 10 meses después de la siembra y se determinó la productividad (kg/planta). Se realizó un análisis de varianza y prueba de Scott-Knott para la comparación de promedios. Se verificó que hubo respuesta creciente a la fertilización fosfatada; la productividad fue mayor para la dosis de $160 \mathrm{~kg}$ de P2O5/ha, que es el doble de la dosis máxima recomendada por la CFSEMG, demostrando que aunque rústica, la planta es responsiva a la fertilización fosfatada. Con respecto a la fertilización de cobertura, no hubo diferencia significativa entre los tratamientos. Se concluye que el cultivo de yuca IAC 12 es responsiva a fósforo, habiendo necesidad de estudios con dosis mayores.
\end{abstract}

Palabras clave: Fertilización de plantio, IAC 12, Manihot esculenta

\section{Introdução}

Mandioca, aipim, macaxeira, ou mandiocabrava são alguns termos brasileiros usados para designar a espécie Manihot esculenta; planta considerada genuinamente brasileira que foi domesticada pelos indígenas antes da descoberta do Brasil. Por se tratar de uma planta bastante rustica e adaptável, o cultivo a mandioca foi aos poucos se popularizando, tornando assim uma das culturas mais plantadas no Brasil, seja para subsistência ou comercialização. De acordo com Lima et al. (2005), a utilização da raiz de mandioca tem destaque na alimentação humana e animal, também é usada na produção de perfumes e fármacos, produtos de limpeza, combustíveis, adesivo têxtil, papel, celulose, explosivos, calcados, tintas e outros, agregando valor econômico e social na geração de emprego.

Por se tratar de uma cultura de fácil adaptação às condições do cerrado, baixo custo e elevado potencial produtivo, a mandioca se constitui em uma das mais promissoras culturas. Originária do continente americano, provavelmente do Brasil Central, a mandioca já era amplamente cultivada pelos indígenas, sendo eles os responsáveis por sua disseminação em quase toda a América e os portugueses e espanhóis pela sua difusão para outros continentes, especialmente África e Ásia. Embora já existam implementos motomecanizados de fabricação nacional, a colheita da mandioca é primordialmente manual.
Em trabalhos com a cultivar Cardoso et al. (2005) citam que a melhor dosagem para adubação fosfatada é de $100 \mathrm{~kg}$ de $\mathrm{P}_{2} \mathrm{O}_{5} /$ ha, e complementa que, a saturação de bases para melhor resposta em conjunto com a dose citada de fósforo é de $25 \%$. Em estudo Devide et al. (2009) trabalharam com a variedade IAC (576-70) em consorcio com a cultura do milho obtendo ótimos resultados, em trabalhos semelhantes Mattos et al. (2005) trabalharam com a mandioca em consórcio com outras culturas e obteve excelentes resultados.

\section{Material e Métodos}

$\mathrm{O}$ experimento foi realizado na cidade de Americano do Brasil, GO, no período de 08/2016 a $11 / 2017$, (latitude: $16^{\circ} 15^{\prime} 17^{\prime}$ ' S; longitude: $49^{\circ}$ 58 ' $58^{\prime}$ ' W) situada a uma altitude de $890 \mathrm{~m}$, foi retirada amostras do solo, de 0 a 20 e 20 a 40 no dia 25/08/2016 e feito análise química. O experimento foi instalado em delineamento experimental em parcela subdividida com 3 repetições, e desenho experimental inteiramente casualizado. Cada parcela (repetição) foi constituída de 3 linhas com 10m de comprimento.

O plantio e adubação fosfatada foram realizados no dia 12/12/2016, utilizando plantadeira de 2 linhas, avaliaram-se quatro doses de fósforo, $\left(0 ; 40 ; 80 ; 160 \mathrm{~kg}\right.$ de $\left.\mathrm{P}_{2} \mathrm{O}_{5} / \mathrm{ha}\right)$. A dose de $80 \mathrm{~kg}$ de $\mathrm{P}_{2} \mathrm{O}_{5} /$ há foi definia conforme análise de solo (Tabela 1) e a recomendação da Ribeiro et al. (1999). 
Tabela 1. Análise de solo da área do experimento. Americano do Brasil, 2017.

\begin{tabular}{|c|c|c|c|c|c|c|c|c|c|c|c|c|}
\hline$\overline{\text { Prof }}$ & $\mathrm{pH}$ & M.O & $\mathrm{P}$ & $\mathrm{K}$ & $\mathrm{Ca}$ & $\mathrm{Mg}$ & $\mathrm{Al}$ & $\mathrm{H}+\mathrm{Al}$ & CTC & $\mathrm{m}$ & Arg & $\mathrm{V}$ \\
\hline$\overline{\mathrm{Cm}}$ & & $\%$ & \multicolumn{2}{|c|}{$\mathrm{mg} / \mathrm{dm}^{3}$} & \multicolumn{4}{|c|}{ cmolc/dm ${ }^{3}$} & \multicolumn{4}{|c|}{$\%$} \\
\hline$\overline{00-20}$ & 5,2 & 0,70 & 1,44 & 46,0 & 0,88 & 0,13 & 0,10 & 2,10 & 3,23 & 8,16 & 26 & 34,6 \\
\hline $20-40$ & 5,2 & 0,60 & 0,81 & 20,0 & 0,63 & 0,50 & 0,15 & 2,10 & 3,28 & 11,2 & 30 & 35,8 \\
\hline
\end{tabular}

O espaçamento do plantio foi de 0,90 metros entre linhas e 0,60 metros entre plantas, após o plantio foi aplicado um combo de herbicida, utilizando (coronel, dual e roundup), para o controle das plantas daninhas. A adubação de cobertura foi realizada no dia 19/02/2017 que avaliou (0 e $250 \mathrm{~kg} / \mathrm{ha}$ do adubo NPK 20-00-20). Segue abaixo descrição dos tratamentos analisados:

- Parcela Principal (Adubação de plantio, com quatro doses):

1) $0 \mathrm{~kg} / \mathrm{ha}$ de fosforo

2) $40 \mathrm{~kg} / \mathrm{ha} \mathrm{de}$ fosforo

3) $80 \mathrm{~kg} / \mathrm{ha} \mathrm{de}$ fosforo

4) $160 \mathrm{~kg} / \mathrm{ha}$ de fosforo

- Subparcelas (Adubação de cobertura, com dois fatores):

1) $\mathrm{Sem}$

2) Com $(250 \mathrm{~kg} / \mathrm{ha}$ de $20-00-20)$

A colheita foi realizada manualmente no dia 23/10/2017, ou seja, 10 meses após o plantio. Foi realizada pesagem da massa fresca das raízes e contagem do número de plantas em cada parcela, calculando-se produtividade média por planta. Os dados foram submetidos à análise variância utilizando o Software estatístico R ( 2016).

\section{Resultados e Discussão}

O resultado da produtividade da mandioca IAC 12 sobre às 4 doses de adubação fosfatada é apresentado na tabela 2.

Observando a tabela 2 verificou-se que houve resposta crescente à adubação fosfatada. A produtividade em $\mathrm{kg} /$ planta foi maior para a dose de $160 \mathrm{~kg}$ de $\mathrm{P}_{2} \mathrm{O}_{5} /$ ha, produzindo $1,072 \mathrm{Kg} /$ planta, que corresponderia a cerca de 19 toneladas por hectare, enquanto o tratamento de $80 \mathrm{~kg}$ de $\mathrm{P}_{2} \mathrm{O}_{5} /$ ha produziu cerca de 16 toneladas por hectare, mostrando que embora rústica, a planta é responsiva a adubação fosfatada em solos arenosos do noroeste do Paraná chegou a resultados semelhantes havendo resposta das cultivares testadas até $90 \mathrm{~kg}$ de $\mathrm{P}_{2} \mathrm{O}_{5} /$ há.

Tabela 2. Produtividade da mandioca mediante a 4 níveis diferentes de adubação fosfatada. Americano do Brasil - GO. 23 de novembro de 2017.

\begin{tabular}{lccccc}
\hline Fósforo & $\begin{array}{c}0 \mathrm{~kg} \\
\mathrm{P}_{2} \mathrm{O}_{5} / \mathrm{ha}\end{array}$ & $\begin{array}{c}40 \mathrm{~kg} \\
\mathrm{P}_{2} \mathrm{O}_{5} / \mathrm{ha}\end{array}$ & $\begin{array}{c}80 \mathrm{~kg} \\
\mathrm{P}_{2} \mathrm{O}_{5} / \mathrm{ha}\end{array}$ & $\begin{array}{c}160 \mathrm{~kg} \\
\mathrm{P}_{2} \mathrm{O}_{5} / \mathrm{ha}\end{array}$ & $\mathrm{CV} \%$ \\
\hline $\begin{array}{l}\text { Produtividade } \\
\text { kg/planta }\end{array}$ & $0,603 \mathrm{~d}$ & $0,773 \mathrm{c}$ & $0,905 \mathrm{~b}$ & $1,072 \mathrm{a}$ & 30,50 \\
\hline $\begin{array}{l}\text { *Médias seguidas pela mesma letra não diferiram } \\
\text { significativamente pelo teste de Scott-Knott a 5\%. }\end{array}$
\end{tabular}

Segundo Sousa \& Lobato (2004) a maioria das plantas cultivadas respondem a adubação fosfatada em solos de cerrado, isto se dá porque os solos de cerrado possuem enorme capacidade de fixação do fosforo, deixando uma pequena quantidade disponível para as plantas, principalmente, em solos que ainda não foram adequadamente corrigidos, como o da área experimental e da maioria das plantações de mandioca do país.

Seguem na tabela 3 os resultados de produtividade da mandioca IAC 12 sobre presença e ausência de cobertura com do fertilizante NPK 20-00-20.

Tabela 3. Produtividade da mandioca mediante a presença e ausência de adubação de cobertura 20-00-20. Americano do Brasil - GO. 23 de novembro de 2017.

\begin{tabular}{llll}
\hline Cobertura $20-00-20$ & SEM & COM & CV \% \\
\hline Produtividade, kg/planta & $0,814 \mathrm{a}$ & $0,863 \mathrm{a}$ & 30,50 \\
\hline
\end{tabular}

*Médias seguidas pela mesma letra não diferiram

significativamente pelo teste de Scott-Knott a 5\%.

Com relação à adubação de cobertura, não houve diferença significativa entre os tratamentos, corroborando com Ribeiro et al. (1999) que citam a baixa resposta da mandioca a nitrogênio e potássio.

\section{Conclusão}

Diante dos resultados conclui-se que a cultivar de mandioca IAC 12 é altamente responsiva a adubação fosfatada, apresentado a maior produtividade (19t/ha) com a maior dose testada 
(160kg/ha de $\left.\mathrm{P}_{2} \mathrm{O}_{5}\right)$. Com relação a adubação de cobertura, verificou-se que não houve resposta significativa. Dessa forma, percebe-se a necessidade de novos estudos com maiores doses da adubação fosfatada, bem como, a sua viabilidade econômica.

\section{Referência Bibliográfica}

Cardoso, E.M.R., Ferreira, W.A., Botelho, S.M. \& Poltronieri, M.C., 2005. Fonte alternativa de fertilizante orgânico no cultivo da mandioca, Campo Grande, Mato Grosso do Sul.

Devide, A.C.P., Ribeiro, R.d.L.D., Valle, T.L., Almeida, D.L.d., Castro, C.M.d. \& Feltran, J.C., 2009. Produtividade de raízes de mandioca consorciada com milho e caupi em sistema orgânico. Bragantia 68, 145-153.

Lima, M.B., Mattos, P.L.P., Souza, J.d.S. \& Caldas, R.C., 2005. Aspectos econômicos da mandioca em diferentes espaçamentos em monocultivo e consorciada com caupi e milho. Revista Brasileira de Mandioca, 18, 17-26.

Mattos, P.L.P., Souza, L.S., Souza, J.S. \& Caldas, R.C., 2005. Consorciação da mandioca plantada em fileiras duplas e simples com culturas de ciclo curto. I. mandioca $\mathrm{x}$ caupi $\mathrm{x}$ milho. Revista Brasileira de Mandioca, 18, 2530.

R-Core-Team, 2016. $\mathrm{R}:$ A language and environment for statistical computing, $R$. Foundation for Satatical Computing, Viena.

Ribeiro, A.C., Guimarães, P.T.G. \& Alvarez, V.H., 1999. Comissão de fertilidade do solo do Estado de Minas Gerais, In: Viçosa, U.F.d. (Ed.), Recomendações para o uso de corretivos e fertilizantes em Minas Gerais, Viçosa, Minas Gerais.

Sousa, D. \& Lobato, E., 2004. Cerrado: correção do solo e adubação. Embrapa Cerrados 1, 416.

Recebido: 18 Abr. 2018

Aprovado: 16 Mai. 2018

Publicado: 27 Jul. 2018

Licenciamento: Este artigo é publicado na modalidade Acesso Aberto sob a licença Creative Commons Atribuição 4.0 (CC-BY 4.0), a qual permite uso irrestrito, distribuição, reprodução em qualquer meio, desde que o autor e a fonte sejam devidamente creditados 